Pacific Journal of Mathematics

ON THE CONVERGENCE OF ASYMPTOTIC SOLUTIONS OF 


\title{
ON THE CONVERGENCE OF ASYMPTOTIC SOLUTIONS OF LINEAR DIFFERENTIAL EQUATIONS
}

\author{
R. M. REDHEFFER and W. WASOW
}

1. Introduction. In the differential equation

$$
L[y, \varepsilon] \equiv y^{(n)}+\sum_{j=1}^{n} A_{j}(x, \varepsilon) y^{(n-j)}=0
$$

let the coefficients $A_{j}(x, \varepsilon)$ be analytic functions of $x$ and $\varepsilon$. For all values of $x$ and $\varepsilon$ for which these coefficients are holomorphic in both variables the differential equation admits a fundamental system of solutions with the same property. But if some coefficients of (1) have poles, as functions of $\varepsilon$, for a certain value of $\varepsilon$, say for $\varepsilon=0$, then the solutions of the differential equation will in general have singularities, as functions of $\varepsilon$, at $\varepsilon=0$. The purpose of this paper is to collect some observations on the question of when solutions holomorphic at $\varepsilon=0$ exist even in this case.

The theory of asymptotic integration of such differential equations [6], [8], [3], [9], [10] teaches that in this case there exist fundamental solutions which are asymptotically represented by generally divergent expansions of the form

$$
e^{P(x, \varepsilon)} \sum_{\nu=0}^{\infty} y_{\nu}(x) \varepsilon^{\nu i r},
$$

where $r$ is a positive integer and $P(x, \varepsilon)$ is a polynomial in $\varepsilon^{-1 / r}$. Our problem might naturally be generalized to include the question of the convergence of any, or all, of these asymptotic series, whether $P(x, \varepsilon)$ be identically zero or not. But this will not be done here.

The analogous problem for differential equations without a parameter, at a point where the coefficients have a singularity has been quite thoroughly investigated (cf. [1, 486-489]). By contrast, there seem to exist no studies of corresponding questions for the dependence on a parameter, nor does it seem possible to transfer the results obtained for one problem to the other by an easy analogy. In view of this situation the results of this paper may be of some interest.

2. Necessary conditions. Let us assume that $A_{j}(x, \varepsilon)$ are of the form

$$
A_{j}(x, \varepsilon)=\varepsilon^{-h} \sum_{k=0}^{\infty} A_{j k}(x) \varepsilon^{k}, \quad(j=1, \cdots, n)
$$

Received December 3, 1953. 
where at least one $A_{j 0}(x)$ is not identically zero and $h$ is a positive integer. The series are supposed to converge when $x$ is in a fixed region $X$ of the $x$-plane, and for $\varepsilon$ in a circle $E:|\varepsilon| \leq \varepsilon_{0}$, $\varepsilon_{0}$ being independent of $x$. The functions $A_{j k}(x)$ are to be holomorphic in $X$. In order to shorten the terminology the self-explanatory expressions $x$-holomorphic and $\varepsilon$-holomorphic will sometimes be used. A function that is holomorphic in both variables may be called $(x, \varepsilon)$-holomorphic.

The differential equation (1) can be rewritten in the from

$$
\varepsilon^{h} L[y, \varepsilon] \equiv \varepsilon^{h} N[y, \varepsilon]+M[y, \varepsilon]=0 \text {. }
$$

Here,

$$
\begin{aligned}
& N[y, \varepsilon]=y^{(n)}+\sum_{\nu=1}^{n} a_{\nu}(x, \varepsilon) y^{(n-\nu)} \\
& M[y, \varepsilon]=\sum_{\mu=0}^{m} b_{\mu}(x, \varepsilon) y^{(m-\mu)},
\end{aligned}
$$

and the $a_{\nu}(x, \varepsilon)$ and $b_{\mu}(x, \varepsilon)$ are $(x, \varepsilon)$-holomorphic in the product space of $X$ and $E$. The $b_{\mu}(x, \varepsilon)$ are polynomials in $\varepsilon$ of degree less than $h$. The coefficient $b_{0}(x, 0)$ is not identically zero. Furthermore, $0 \leq m<n$. By formal substitution of a power series $\sum_{j=0}^{\infty} y_{j}(x) \varepsilon^{j}$ into (4) it is seen that nontrivial formal power series solutions can be constructed if, and only if,

$$
m>0 \text {. }
$$

If (5) is satisfied, then the function $y_{0}(x)$ may be any solution of the "reduced" differential equation

$$
M[y, 0]=\sum_{\mu=0}^{m} b_{\mu}(x, 0) y^{(m-\mu)}=0,
$$

and the functions $y_{j}(x), j \geq 1$ can be successively calculated, in infinitely many ways, as solutions of a sequence of nonhomogeneous differential equations whose homogeneous part is $M\left[y_{j}, 0\right]$.

Let us call a solution which is $\varepsilon$-holomorphic at $\varepsilon=0$, a regular solution. Unless it is important in the context, we shall not specify the $x$-domain for which such a solution is regular. A set of regular solutions will be simply called independent, if the solutions are linearly independent at $\varepsilon=0$, and hence in some neighborhood of $\varepsilon=0$. From the preceding discussion it follows that the differential equation (1) cannot have more than $m$ independent regular solutions.

It is easy to construct examples for which the number of independent regular solutions is equal to $m$. Let, for instance, $Y_{j}(x, \varepsilon)$, $(j=1, \cdots, m)$ be $m$ linearly independent functions that are $(x, \varepsilon)$-holomorphic in the product space of $X$ and $E$, and denote by $M[y, \varepsilon]=0$ 
the linear differential equation of order $m$ with leading coefficient one that is satisfied by these functions. If $D$ designates the operation of differentiation with respect to $x$, then

$$
\varepsilon^{n-m} D^{n-m} M[y, \varepsilon]+M[y, \varepsilon]=0,
$$

$n>m$

is an $n$th order differential equation with $m$ regular solutions. The standard asymptotic theory (see e.g. [9]) shows that the functions $Y_{j}(x, \varepsilon)$ are part of a fundamental system of (7) whose $n-m$ remaining solutions have asymptotic representations of the form (2) with $P(x, \varepsilon)$ equal to the $n-m$ determinations of $(-1)^{1 /(n-m)} \varepsilon^{-1} x$.

In spite of this, the occurrence of any regular solution must be regarded as exceptional. In order to show this we prove the following lemma, which generalizes a result of Horn [2].

LEMMA 1. Let the coefficients $\alpha_{j \mu}(x, \varepsilon)$ of the system of differential equations

$$
\frac{d u_{j}}{d x}=\varepsilon^{-h} \sum_{\mu=1}^{n} \alpha_{j \mu}(x, \varepsilon) u_{\mu}, \quad(j=1, \cdots, n)
$$

be $(x, \varepsilon)$-holomorphic for $x$ in $X$ and for $|\varepsilon| \leq \varepsilon_{0}$. Let the solution $u_{j}=U_{j}(x, \varepsilon)$ of $(8)$ be characterized by the initial values

$$
U_{j}(a, \varepsilon)=p_{j}(\varepsilon)
$$$$
(j=1, \cdots, n)
$$

at a point $a$ of $X$, where the functions $p_{j}(\varepsilon)$ are holomorphic for $|\varepsilon| \leq \varepsilon_{0}$, except possibly for a pole at $\varepsilon=0$. Then

$$
U_{j}(x, \varepsilon)=U_{j}^{*}(x, \varepsilon)+U_{j}^{* *}\left(x, \frac{1}{\varepsilon}\right)
$$

where $U_{j}^{*}, U_{j}^{* *}$ are $x$-holomorphic in $X$, and $\varepsilon$-holomorphic for $|\varepsilon| \leq \varepsilon_{0}$ and $|\varepsilon| \geq 0$, respectively.

Proof. Define the functions $U_{\jmath r}(x, \varepsilon)$ by the relations

$$
U_{j r}(x, \varepsilon)= \begin{cases}p_{j}(\varepsilon), & r=0 \\ \varepsilon^{-l} \int_{\Gamma_{\alpha x}} \sum_{\mu=1}^{n} \alpha_{j \mu}(t, \varepsilon) U_{j, r-1}(t, \varepsilon) d t, & r>0\end{cases}
$$

where $I_{a x}$ is a path connecting $a$ and $x$ in $X$. By the standard argument of Picard's iteration method it follows that for $0<\varepsilon_{1} \leq|\varepsilon| \leq \varepsilon_{0}$, and for $x$ in any closed and bounded subdomain of $X$,

$$
U_{j}(x, \varepsilon)=\sum_{r=0}^{\infty} U_{j r}(x, \varepsilon)
$$

where the series, as well as the series of its termwise derivatives with respect to $x$, converge uniformly and absolutely in the indicated domain. If $k$ is the highest order of the poles of the functions $p_{j}(\varepsilon)$, 
the formulas (10) show that the iterants are of the form

$$
U_{j r}(x, \varepsilon)=\varepsilon^{-(r h+k)} V_{j r}(x, \varepsilon)
$$

where the $V_{j r}(x, \varepsilon)$ are $(x, \varepsilon)$-holomorphic for $x$ in $X$ and $|\varepsilon| \leq \varepsilon_{0}$. From (11) and (12) we conclude, by means of Weierstrass's theorem on interchange of summations in double series that $U_{j}(x, \varepsilon)$ admits a convergent representation of the form

$$
U_{j}(x, \varepsilon)=\sum_{\nu=-\infty}^{\infty} F_{j \nu}(x) \varepsilon^{\nu},
$$

which is uniformly valid for $x$ in every closed subdomain of $X$, and for $0<\varepsilon_{1} \leq|\varepsilon| \leq \varepsilon_{0}$, where $\varepsilon_{1}$ is arbitrary, and the $F_{j \nu}(x)$ are holomorphic in $X$. This proves the lemma.

Suppose, now, that the differential equation (1) admits a regular solution $Y(x, \varepsilon)$ in some subdomain $X^{*}$ of $X$. If $a$ is any point of $X^{*}$, then $Y(x, \varepsilon)$ can be uniquely characterized by the values of $Y^{(s)}(\alpha, \varepsilon)$, $(s=0, \cdots, n-1)$, which are $\varepsilon$-holomorphic for $|\varepsilon| \leq \varepsilon_{0}$. Since the differential equation (1) is equivalent to a system of the form (8), it follows from the lemma just proved that

$$
Y(x, \varepsilon)=\phi_{1}(x, \varepsilon)+\phi_{2}(x, \varepsilon),
$$

$x \in X$,

where $\phi_{1}, \phi_{2}$ are $\varepsilon$-holomorphic in $|\varepsilon| \leq \varepsilon_{0}$, and $|\varepsilon|>0$, respectively, and $x$-holomorphic in $X$. But since $Y(x, \varepsilon)$ is $\varepsilon$-holomorphic for $|\varepsilon| \leq \varepsilon_{0}$ and $x$ in $X^{*}$, the uniqueness theorem for Laurent's expansion leads to the conclusion that $\phi_{2}(x, \varepsilon) \equiv 0$ for $x$ in $X^{*}$ and all $\varepsilon$. Being $x$-holomorphic in $X$ by Lemma $1, \phi_{:}(x, \varepsilon)$ vanishes therefore identically in the whole domain $X$. This implies, in particular, that $Y(x, 0)$ is $x$-holomorphic in $X$. On the other hand, $Y(x, 0)$ is a solution of the reduced equation $M[y, 0]=0$, and we have proved the following theorem.

THEOREM 1. If the full differential equation (4) possesses a regular solution $Y(x, \varepsilon)$, then the corresponding solution $Y(x, 0)$ of the reduced equation $M[y, 0]=0$ must be $x$-holomorphic in every domain $X$ where the coefficients of the full equation are x-holomorphic.

This is a rather strong restriction on the coefficients of $M[y, 0]=0$, in particular on $b_{0}(x)$. For the equation $M[y, 0]=0$ has, in general, singularities at all zeros of $b_{0}(x)$, and there will rarely exist a solution of $M[y, 0]=0$ that is holomorphic at all the zeros of $b_{0}(x)$ which lie in $X$.

Theorem 1 sheds some light on Theorem 9.2 of [11]. That paper was concerned with the special case in which the expression $N[y, \varepsilon]$ $\equiv N[y]$ of (4) was of order four and independent of $\varepsilon$, and $M[y, \varepsilon]$ was of the form

$$
M[y] \equiv b_{0}(x) y^{\prime \prime}+b_{2}(x) y
$$


where $b_{0}(x)$ had a first-order zero at $x=0$. In Theorem 9.2 it was proved that the full equation

$$
\varepsilon N[y]+M[y]=0
$$

possesses in this case a solution $y=V(x, \varepsilon)$ that approaches, uniformly in a full neighborhood $X^{*}$ of $x=0$, the $x$-holomorphic solution $v(x)$ of $M[y]=0$, as $\varepsilon \rightarrow 0$ along a given ray of the $\varepsilon$-plane. It would seem plausible to conjecture that $V(x, \varepsilon)$ is $\varepsilon$-holomorphic at $\varepsilon=0$. But Theorem 1 shows that this is, in general, not the case, at least, if $b_{0}(x)$ possesses other zeros besides $x=0$.

3. Some remarks on sufficient conditions for convergence. The problem of finding sufficient conditions for the convergence of an asymptotic series in $\varepsilon$ seems to be much more difficult than the topic discussed in the preceding section but some special classes of differential equations admitting regular solution can be constructed.

a) Constant coefficients. If the coefficients of the differential equation (4) are independent of $x$ it possesses a solution of the form $y=e^{\lambda(\varepsilon) x}$ corresponding to every distinct root $\lambda(\varepsilon)$ of the polynomial equation

$$
H(\lambda, \varepsilon) \equiv \varepsilon^{n}\left\{\lambda^{n}+\sum_{\nu=1}^{n} a_{\nu}(\varepsilon) \lambda^{n-\nu}\right\}+\sum_{\mu=0}^{m} b_{\mu}(\varepsilon) \lambda^{m-\mu}=0 .
$$

Let $\lambda=\lambda_{0}$ be a root of the equation

$$
H(\lambda, 0) \equiv \sum_{\mu=0}^{m} b_{\mu}(0) \lambda^{m-\mu}=0,
$$

then by classical implicit function theorems $H(\lambda, \varepsilon)$ possesses an $\varepsilon$-holomorphic root for which $\lambda(0)=\lambda_{0}$, provided $\partial H / \partial \lambda$ does not vanish for $\varepsilon=0, \lambda=\lambda_{0}$, that is, provided $\lambda_{0}$ is a simple root of $H(\lambda, 0)$. If all roots of $H(\lambda, 0)$ are multiple, $H(\lambda, \varepsilon)=0$ may or may not define an $\varepsilon$-holomorphic function, as can be seen from the example

$$
H(\lambda, \varepsilon) \equiv \varepsilon^{n} \lambda^{3}+\lambda^{2}-2 \lambda+1=0,
$$

which possesses an $\varepsilon$-holomorphic solution for $h=2$, but not for $h=1$.

b) Linear coefficients. In formulas (4a) and (4b) let

$$
\begin{aligned}
& a_{\nu}(x, \varepsilon)=a_{0 \nu}(\varepsilon)+a_{1 \nu}(\varepsilon) x \\
& b_{\mu}(x, \varepsilon)=b_{0 \mu}(\varepsilon)+b_{1 \mu}(\varepsilon) x .
\end{aligned}
$$

For many differential equations of this type regular solutions can be found by means of complex Laplace transformation. If we introduce the polynomials 


$$
A_{j}=A_{j}(t, \varepsilon)=\sum_{\nu=1}^{n} a_{j \nu}(\varepsilon) t^{n-\nu}
$$

$$
B_{j}=B_{j}(t, \varepsilon)=\sum_{\mu=0}^{m} b_{j \mu}(\varepsilon) t^{m-\mu},
$$

then the differential equation (4) with coefficients of the form (14) admits $[4, \S \S 8,18]$ solutions of the form

$$
y(x, \varepsilon)=\int_{C} v(t, \varepsilon) e^{t x} d t
$$

where $v(t, \varepsilon)$ is a solution of the differential equation

$$
\left(\varepsilon^{n} t^{n}+\varepsilon^{n} A_{0}+B_{0}\right) v-\frac{d}{d t}\left[\left(\varepsilon^{n} A_{1}+B_{1}\right) v\right]=0
$$

and $C$ a suitable contour.

If a closed contour $C$ on the Riemann surface of $v(t, \varepsilon)$ as a function of $t$ can be found such that $C$ is independent of $\varepsilon$ and the integral (16) exists for all small $\varepsilon$, then the integral will be either zero or furnish a regular solution, since $C$ can then be chosen so as to avoid the points where $v(t, \varepsilon)$ is not $\varepsilon$-holomorphic. It is possible, but not very illuminating, to formulate more explicit sufficient conditions on the coefficients under which the preceding condition can be satisfied. Some special differential equations of this type were treated in [12] and [13]. The equations

$$
\begin{aligned}
& \varepsilon y^{(4)}+x y^{\prime \prime}+y=0 \\
& \varepsilon y^{(4)}+x\left(y^{\prime \prime}+y\right)=0
\end{aligned}
$$

do possess regular solutions. The differential equation

$$
\varepsilon y^{\prime \prime}+k x y^{\prime}+y=0
$$

turns out to have a regular solution when the constant $1 / k$ is a negative integer. For other values of $k$ the solution of the reduced equation has a singular point at $x=0$ and the sole regular solution is $y \equiv 0$, in consequence of Theorem 1 .

4. The differential equation $\varepsilon y^{\prime \prime}+a(x) y^{\prime}+b(x) y=0$.

a) Polynomial initial conditions. The theorem of $\S 2$ suggests the conjecture that regular solutions exist if the coefficients of the differential equation are entire functions without zeros. But the example $\varepsilon y^{\prime \prime \prime}+y^{\prime \prime}-2 y^{\prime}+y=0$ mentioned in the preceding section shows that this conjecture is certainly not true in full generality. In this section some sufficient conditions are established for regularity, attention being confined to the equation $\varepsilon y^{\prime \prime}+a(x) y^{\prime}+b(x) y=0$. In agreement 
with the foregoing results, the conditions on $a(x)$ and $b(x)$ take account of the behavior in the large. For example, a solution $x$-holomorphic for $|\varepsilon|<\varepsilon_{0},|x|<x_{0}$ must be in fact $x$-holomorphic for $\varepsilon=0$, whenever $x \in X$, the domain of regularity of $a$ and $b$. A hypothesis ensuring regularity must therefore ensure, at least implicitly, that the reduced equation $a y^{\prime}+b y=0$ has a solution of the indicated type.

We consider the following statements:

Statement (A). The equation $\varepsilon y^{\prime \prime}+a(x) y^{\prime}+b(x) y=0$ has a solution

$$
y(x, \varepsilon)=\sum_{n=0}^{\infty} y_{n}(x) \varepsilon^{n} \rightleftharpoons 0
$$

convergent for $|\varepsilon|<\varepsilon_{0}, \quad|x|<x$, and satisfying $y_{n}(0)=P(n), y_{n}^{\prime}(0)=Q(n)$, where $P(n)$ and $Q(n)$ are polynomials of degree $\leq k$.

Statement (B). The equation admits a regular solution $y(x, \varepsilon)$ such that $y_{x}(0, \varepsilon) / y(0, \varepsilon)$ is a rational function of $\varepsilon$, whose numerator and denominator have degree $\leq k$.

Statement (C). The equation admits a solution of the form

$$
\sum_{i=0}^{k} h_{i}(x) \varepsilon^{i \doteq 0}=0,
$$

where the $h$ 's are holomorphic near $x=0$.

Statement (D). We have $H^{k}[f(x)] \equiv 0$ for a linear function $f(x) \equiv 0$, where the operator $H$ is defined by

$$
H(p)=p+\int_{0}^{x} a p d x+\int_{0}^{x} \int_{0}^{x_{1}}\left(b-a^{\prime}\right) p d x d x_{1} .
$$

It will be shown, now, that these statements are closely related, $a(x)$ and $b(x)$ being holomorphic near $x=0$ :

THEOREM 2. Statements (A) and (C) are equivalent; (B) is equivalent to them provided $a(0) \neq 0$; and (D) implies all three.

To establish the theorem, suppose Statement (A) given, and equate coefficients to find

$$
-y_{n-1}^{\prime \prime}=a y_{n}^{\prime}+b y_{n}, \quad \text { for } n \geq 1, \quad 0=a y_{0}^{\prime}+b y_{0},
$$

which becomes

$$
\begin{aligned}
y_{n-1}^{\prime}-Q(n-1) & =\int_{0}^{x}\left(a y_{n}^{\prime}+b y_{n}\right) d x=-\left.a y_{n}\right|_{0} ^{x}+\int_{0}^{x} y_{n}\left(b-a^{\prime}\right) d x \\
& =-a y_{n}+a(0) P(n)+\int_{0}^{x} y_{n}\left(b-a^{\prime}\right) d x .
\end{aligned}
$$

Further integration yields

$$
y_{n-1}-P(n-1)-x Q(n-1)=\int_{0}^{x} \int_{0}^{x_{1}} y_{n}\left(b-a^{\prime}\right) d x d x_{1}-\int_{0}^{x} a y_{n} d x+a(0) P(n) x
$$


Let $Y_{n}=\Delta^{k+1} y_{n}$, the $(k+1)$ th difference. We have, by $(22)$,

$$
Y_{n-1}=\int_{0}^{x} \int_{0}^{x_{1}} Y_{n}\left(b-a^{\prime}\right) d x d x_{1}-\int_{0}^{x} a Y_{n} d x .
$$

Regularity of $a$ and $b$, convergence of $\sum y_{n} \varepsilon^{n}$, ensure that for $|x| \leq x^{*}<x_{0}$ we have

$$
\left|Y_{n}\right| \leq B A^{n}, \quad|a| \leq M, \quad\left|a^{\prime}\right| \leq M, \quad|b| \leq M,
$$

where $A, B$, and $M$ are suitable positive constants (that may depend on $x^{*}$ ). Thus, we have by (23) in every circle $|x| \leq \delta<1$, with $\delta \leq x^{*}$,

$$
\max \left|Y_{n-1}\right| \leq 3 \delta M\left(\max \left|Y_{n}\right|\right) \leq(2 \delta M)^{m+1}\left(\max \left|Y_{n+m}\right|\right), \quad m=0,1, \cdots
$$

the latter relation following by iteration. Choose $\delta$ so small that $4 \delta M<1 / A$. Then

$$
\left|Y_{n-1}\right|<B(1 / 2 A)^{m+1} A^{n+m}=B A^{n-1} / 2^{m+1} .
$$

Letting $m \rightarrow \infty$ shows that $\left|Y_{n}\right|=0$, and hence

$$
y_{n}=g_{0}(x)+g_{1}(x) n+g_{2}(x) n^{2}+\cdots+g_{k}(x) n^{k} .
$$

It follows that $y(x, \varepsilon)$ has the form

$$
y(x, \varepsilon)=\sum_{i=0}^{k} \frac{f_{i}(x)}{(1-\varepsilon)^{i+1}}
$$

as we see by using factorial polynomials in place of powers of $n$. Multiplying through by $(1-\varepsilon)^{k+1}$ shows that (A) implies (C). ${ }^{1}$

To see that (C) implies (A), express the given polynomial as a new polynomial in $1-\varepsilon$ and divide by $(1-\varepsilon)^{k+1}$. We are led to a solution of the form (24), and expansion of $(1-\varepsilon)^{-i-1}$ gives the initial conditions described in (A). We have incidentally established the rather curious fact that $y_{n}(x)$ and $y_{n}^{\prime}(x)$ are polynomials in $n$ for every small fixed $x$, if for the single value $x=0$.

Suppose now that (C) is given. We may assume (24). With $s=1 /(1-\varepsilon)$, equating powers of $s$ in $\varepsilon y^{\prime \prime}+a y^{\prime}+b y=0$ gives

$$
\left\{\begin{array}{l}
f_{0}^{\prime \prime}=0 \\
f_{1}^{\prime \prime}=L\left(f_{0}\right) \\
f_{2}^{\prime \prime}=L\left(f_{1}\right) \\
\cdots \cdots \cdots \\
f_{k}^{\prime \prime}=L\left(f_{k-1}\right) \\
0=L\left(f_{k}^{\prime \prime}\right)
\end{array}\right\}, \quad L(y)=y^{\prime \prime}+a y^{\prime}+b y,
$$

and conversely, the system (25) for some $f_{i} 0$ ensures a solution of

1 A simpler proof has been given by Robert Steinberg, starting with the observation that $(1-\varepsilon)^{k} y(x, \varepsilon)=\sum Y_{n}(x) \varepsilon^{n}$ has $Y_{n}(0)=Y_{n}^{\prime}(0)=0$. 
the form (24), hence of the form described in Statement (C).

We have $\frac{d^{\prime}}{d x^{2}} H(p)=L(p)$, and hence, when the constants of integration are taken as zero in (25), this system is equivalent to

$$
\left\{\begin{array}{c}
f_{0}^{\prime \prime}=0 \\
f_{1}=H\left(f_{0}\right) \\
f_{2}=H\left(f_{1}\right) \\
\cdots \cdots \cdots \\
f_{k}=H\left(f_{k-1}\right) \\
0=H\left(f_{k}\right) .
\end{array}\right.
$$

Hence $H^{k} f_{0}=0$ is sufficient to ensure a solution of (25), and indeed with $f_{i}^{\prime}(0)=f_{i}(0)=0$ for $i \geq 1$. Thus, (D) implies (C), and hence (D) implies (A). The converse is false; but if we define

$$
H(p, q) f \equiv H f+p x+q
$$

Statement $(\mathrm{A})$ or $(\mathrm{C})$ is equivalent to

$$
\stackrel{i}{i=1}_{i} H\left(p_{i}, q_{i}\right) f \equiv 0, \quad f=c x+d \rightleftharpoons 0,
$$

for some constants $p_{i}, q_{i}$. Here $f$ is the first function $f_{i}$ in (25) which is not identically zero.

If (B) is given, suppose $y(0, \varepsilon)$ has a zero of order $h>0$ at $\varepsilon=0$. Then $y_{0}(0)=y_{1}(0)=\cdots=y_{n-1}(0)=0$. The system $(20)$ gives $y_{0}=c \exp \left[-\int_{0}^{x}(b / a) d x\right]$ where $c$ is constant. If $a(0) \rightleftharpoons 0$, it follows that $y_{0}(x) \equiv 0$ for small $x$ and hence for all $x € X$. Similarly, $y_{1}, \cdots, y_{h-1} \equiv 0$ for small $x$. Hence the function $\varepsilon^{-h} y(x, \varepsilon)$ is $\varepsilon$-holomorphic for $\varepsilon=0$ and small $x$.

If $y_{x}(0, \varepsilon) / y(0, \varepsilon)=P(\varepsilon) / Q(\varepsilon)$, where $P$ and $Q$ have degree $\leq k$, then the function $\varepsilon^{-h} y(x, \varepsilon)$ satisfies the same condition. Combining this observation with the preceding, we see that one may suppose $y(0,0) \rightleftharpoons 0$ in Statement $(\mathrm{B})$, provided $a(0) \rightleftharpoons 0$.

Putting $t=1-\varepsilon$, dividing numerator and denominator by $t^{k+1}$, and relabeling coefficients, transforms the given condition into

$$
\begin{gathered}
y_{x}(0, \varepsilon) \\
y(0, \varepsilon)
\end{gathered}=\frac{A(\varepsilon)}{B(\varepsilon)}
$$

where

$$
A(\varepsilon)=a_{0}(1-\varepsilon)^{-1}+a_{1}(1-\varepsilon)^{-2}+\cdots+a_{k}(1-\varepsilon)^{-k-1},
$$

and similarly for $B(\varepsilon)$. The function 


$$
Y(x, \varepsilon)=\frac{y(x, \varepsilon)}{y(0, \varepsilon)} B(\varepsilon)
$$

is regular near $\varepsilon=0$; it satisfies the given differential equation; and also $Y(0, \varepsilon)=B(\varepsilon), \quad Y_{x}(0, \varepsilon)=A(\varepsilon)$. Hence $Y(x, \varepsilon)$ satisfies the requirements of Statement (A).

Finally, it is clear that (B) follows from (C) if $y(0, \varepsilon) \doteq 0$ in (C). If $y(0, \varepsilon) \equiv 0$, however, we have $y_{n}(0)=0$ in $(20)$, which implies $y_{n}(x) \equiv 0$ for $a(0) \rightleftharpoons 0$ as above. Hence $y(x, \varepsilon) \equiv 0$ contrary to the assumption in Statement (C).

The condition on the operator $H$ admits a simple interpretation. If $I$ is the identity operator, then formally

$$
(I-H z)^{-1} p=\sum_{i=0}^{\infty} z^{i} H^{i} p, \quad H^{0}=I .
$$

Now, when $H^{i}(c x+d)=0$ for $i \geq k$, as in Statement (D), then the above expression is a polynomial in $z$ for $p=c x+d$. Suppose, more generally, that

$$
(I-H z)^{-1}(c x+d)=\phi(z, x),
$$

a function holomorphic in $z$ at $z=1$. Then $c x+d=(I-H z) \phi$ or, by differentiating,

$$
0=(1-z) \phi^{\prime \prime}-z a \phi^{\prime}-z b \phi .
$$

With $\varepsilon=1-1 / z$ this yields

$$
\varepsilon \phi^{\prime \prime}+a \phi^{\prime}+b \phi=0
$$

where $\phi$ is $\varepsilon$-holomorphic near $\varepsilon=0$.

The above treatment is purely formal. If

$$
\lim \left|H^{k} p\right|^{1 / k} \leq \theta<1
$$

for $p=c x+d$ and $|x|<\delta$, however, then the formal equalities become true equalities. We define $(I-H z)^{-1}$ by the foregoing series, which converges uniformly in $x$ near $z=1$. The function $H^{k} p$ being analytic for each $k$, we may differentiate the series to find that $\phi(z, x)$ is in fact a solution holomorphic in $z$ for $|z|<1 / \theta$. The corresponding domain of $\varepsilon$ is $|1-\varepsilon|>\theta$. Hence a sufficient condition that the equation have a solution $(\varepsilon, x)$-holomorphic for $|1-\varepsilon|>\theta$ and $|x|<\delta$ is that $\lim \left|H^{k} p\right|^{1 / k}$ $\leq \theta$ for $p=c x+d \equiv 0$. An extension can be given after the manner of (27).

b) Examples and discussion. The preceding result enables us to construct equations admitting regular solutions. If the polynomials in Statement (A) are constant, so that $k=0, \quad P(n)=p, Q(n)=q$, then Statement (C) yields $y(x, \varepsilon)=h(x)$. The differential equation shows that 
$h(x)$ is linear, whence $h(x)=p+q x$ by the initial conditions. Such a function is a solution if and only if $a q+b(p+q x)=0$. The differential equation then takes the form

$$
\varepsilon y^{\prime \prime}+b(x)\left[(c-x) y^{\prime}+y\right]=0
$$

where $c$ is constant. For every choice of $b(x)$ there is obviously a regular solution; namely, $y=x-c$.

The case $k=0$ just considered can be regarded in a different light. Let $a(x)$ and $b(x)$ be integrable and satisfy $|a| \leq M,|b| \leq M$ for a domain of the (possibly complex) variable $x$. The Picard iteration procedure shows then that

$$
\varepsilon y^{\prime \prime}+a y^{\prime}+b y=0
$$

has a unique solution $y(x, \varepsilon)$ subject to $y(0, \varepsilon)=c, y^{\prime}(0, \varepsilon)=d$, where $c$ and $d$ are indepenent of $\varepsilon$. Moreover, this solution is an entire function in $1 / \varepsilon$, of exponential type $M$ at most. If we require a solution $Y(x, \varepsilon) \varepsilon$-holomorphic near $\varepsilon=0$ and satisfying the same initial condition, it is necessary that $y \equiv Y$. This shows that both $y$ and $Y$ are $\varepsilon$-holomorphic in the extended $\varepsilon$-plane, hence independent of $\varepsilon$. Thus we are led to the situation found otherwise above. This discussion resembles that used previously for the more general equation (4).

Turning now to the case $k=1$ in Statement (A), we find

$$
\left\{\begin{aligned}
f_{0}^{\prime \prime} & =0 \\
f_{1}^{\prime \prime} & =a f_{0}^{\prime}+b f_{0} \\
0 & =f_{1}^{\prime \prime}+a f_{1}^{\prime}+b f_{1}
\end{aligned}\right.
$$

by (25). Adding the three equations, or considering $y(x, 0)$, we see that $s=f_{0}+f_{1}$ satisfies the reduced equation $a s^{\prime}+b s=0$. Hence, with $c_{0}$ constant,

$$
f_{0}+f_{1}=c_{0} R(x), \quad R(x)=e^{-\int_{0}^{x}(b / a) d x}
$$

where $R$ must be regular since $f_{0}$ and $f_{1}$ are. If $c_{0}=0$ one easily shows that the problem reduces to the case $k=0$ just considered. Without loss of generality, therefore, we may take $c_{0}=1$. In terms of $R$, the original differential equation is

$$
\varepsilon y^{\prime \prime}+R a(x)(y / R)^{\prime}=0
$$

and the system (29) is equivalent to the three conditions (30), $f_{0}=c x+d$ with $c, d$ constant, and

$$
R^{\prime \prime}=[(c x+d) / R]^{\prime} a R .
$$

The differential equation, then, is 


$$
\varepsilon y^{\prime \prime}+\frac{R^{\prime \prime}}{[(c x+\bar{d}) / R]^{\prime}}\left(\frac{y}{R}\right)^{\prime}=0
$$

and the solution is given by Statement (C) as

$$
y(x, \varepsilon)=R-(c x+d) \varepsilon .
$$

That (34) is in fact a solution is easily verified by actual substitution. In summary, there is a regular solution, with $y_{n}(0)$ and $y_{n}^{\prime}(0)$ linear functions of $n$, if and only if the equation can be put in the form (33); and the sole such solution is then a constant multiple of (34), divided by $(1-\varepsilon)^{2}$.

The case $k=2$ is more complicated. It is found that $a(x)$ must satisfy a certain first-order nonlinear differential equation, $R$ being given, and the case corresponding to $c_{0}=0$ in (30) reduces to the case $k=1$. It would be desirable to find an explicit form of the equation for $k \geq 2$, but we have not been able to do this.

Although the foregoing considerations restrict the behavior of $a$ and $b$ in the large (by virtue of analytic continuation) the analyticity of $a$ and $b$ plays no very essential role. Indeed a corresponding realvariable result might be given, with hypothesis on the local behavior only. It seems difficult to give criteria in which the complex-variable character of the problem is more fully used. This difficulty is illustrated by the following two examples.

Let $a=b$, in the discussion leading to (33) and (34), so that $R=e^{-x}$. If $c=0, d=1$ the differential equation is

$$
\varepsilon y^{\prime \prime}+y^{\prime} e^{-x}+y e^{-x}=0
$$

with solution $y=e^{-x}-\varepsilon$. It is seen that $R, a, b$, and $1 / a$ are entire functions of exponential type, as is the solution $y$.

Consider, next, the equation

$$
\varepsilon y^{\prime \prime}+y^{\prime} e^{x}+y e^{x}=0 .
$$

Despite the resemblance to (35), there is no regular solution, as we now show; and thus the conditions just described, stringent though they be, are yet insufficient.

Suppose there is a regular solution of (36). Since $a(0)=1$ we may assume $y(0,0) \rightleftharpoons 0$, as in the above discussion. The function $y(x, \varepsilon) / y(0, \varepsilon)$ therefore is regular and has $y_{0}(0)=1, y_{n}(0)=0,(n \geq 1)$ in the series representation. The system (20) gives

$$
y_{n}=e^{-x}\left\{c_{n}+y_{n-1}^{\prime}\right\}, \quad y_{0}=e^{-x}
$$

where the $c_{n}$ are constants. By induction we see that 


$$
y_{n}=n ! e^{-(n+1) x}+(n+1) ! c_{1} e^{-n x}+(n-2) ! c_{2} e^{-(n-1) x}+\cdots+c_{n-1} e^{-2 x x}-c_{n} e^{-x}
$$

and, in view of the initial conditions, that $c_{i}>0$. We have therefore

$$
e^{x} y_{n}(x)=e^{x} y_{n}(x)-y_{n}(0)=n !\left(e^{-n x}-1\right)+\cdots, \quad(n \geq 1),
$$

where the terms not written are of the same sign as the leading term, for real $x$, since $c_{n}$ drops out. Thus it is that $\left|y_{n}(x)\right|>n ! e^{-x}\left|e^{-n x}-1\right|$, and the series diverges for $x \neq 0$.

c) Related partial differential equations. Consider the following problems, with regular $a, b$ :

Problem $(A)$. To find a solution $y(x, \varepsilon) \equiv 0$ of $\varepsilon y^{\prime \prime}+a y^{\prime}+b y=0$ which is holomorphic in $\varepsilon$ for $|\varepsilon|<r$, in a given set $S$ of $x$ values.

Problem $(B)$. To find a solution $Y(x, \varepsilon) \neq 0$ of $Y_{x x}+a Y_{x \varepsilon}+b Y_{\varepsilon}=0$ which is an entire function of type $1 / r$ in $\varepsilon$ for a given set $S$ of $x$ values, and satisfies

$$
Y(x, 0)=e^{-\int_{0}^{x}(b \mid a) d x}
$$

or $Y(x, 0)=0$.

It will be shown, now, that these problems are completely equivalent. If

$$
y(x, \varepsilon)=\sum_{0}^{\infty} y_{n}(x) \varepsilon^{n}
$$

is a solution of Problem (A) then

$$
Y(x, \varepsilon)=\sum_{0}^{\infty} y_{n}(x) \varepsilon^{n} / n !
$$

satisfies the differential equation $Y_{x x}+a Y_{x \varepsilon}+b Y_{\varepsilon}=0$. This can be verified by termwise differentiation, insertion into the partial differential equation and use of equations (20). Since $Y(x, 0)=y_{0}(x)$, the first equation in (20) shows that the initial condition of Problem (B) is also statisfied. Finally, it is easy to prove and doubtless well known that $Y$ is an entire function of $\varepsilon$ of type $1 / r$, if and only if $y(x, \varepsilon)$ is $\varepsilon$-holomorphic for $|\varepsilon|<r$.

To show, conversely, that a solution $Y(x, \varepsilon)=\sum z_{n}(x) \varepsilon^{n}$ of Problem (B), leads to a solution of (A) we observe that, by virtue of the statement in the last sentence, the series

$$
y(x, \varepsilon)=\sum z_{n}(x) n ! \varepsilon^{n}
$$

converges. The functions $y_{n}(x)=z_{n}(x) n$ ! are then seen to satisfy the recursion formulas (20) for $n \geq 1$. That they also hold for $n=0$ follows from the initial condition imposed on $y(x, \varepsilon)$. This completes the proof.

We remark in passing that $y$ and $Y$ are transforms of each other: 


$$
\int_{0}^{\infty} e^{-1 t} Y(x, \varepsilon) d \varepsilon=y(x, p)
$$

The change of variable

$$
s=\varepsilon-\int_{0}^{x} a(x) d x, \quad t=\varepsilon
$$

is suggested by the characteristics, and reduces the partial differential equation to canonical form

$$
u_{s t}=\frac{b-a^{\prime}}{a^{2}} u_{s}+\frac{b}{a^{2}} u_{t}
$$

where $u(s, t)=Y(x, \varepsilon)$ and the coefficients are evaluated at $x$. The initial condition is

$$
u\left(-\int_{0}^{x} a(x) d x, 0\right)=e^{-\int_{0}^{x}(b / a) d x}
$$

With $z=e^{-x}-1$ this becomes $u(z, 0)=z+1$ when $a=b=e^{-x}$; but $u(z, 0)$ $=1 /(1-z)$ when $a=b=e^{x}$. Thus the initial values have a pole at $x=0$, in the second case. We have seen already that the solution is regular in the first case but not in the second.

A related partial differential equation arises in another way if we seek a solution $y(x, \varepsilon)$ which is an entire function of type $k$ and such that

$$
\int_{-\infty}^{\infty}|y(x, i \sigma)|^{2} d \sigma<\infty
$$

Such functions are equivalent with those representable in the form

$$
y(x, \varepsilon)=\int_{-k}^{k} e^{\imath} f(x, t) d t, \quad \int_{-k}^{k}|f(x, t)|^{2} d t<\infty .
$$

One obtains, formally,

$$
\int_{-k}^{h}\left(\varepsilon f_{x x}+a f_{x}+b f\right) e^{\varepsilon t} d t=0 .
$$

Integration by parts yields

$$
\int_{-k}^{k} \varepsilon\left(F_{t x x}-a F_{x}-b F\right) d t+e^{\S t}\left[a F_{x}(x, k)+b F(x, k)\right]=0,
$$

where

$$
F(x, t)=\int_{-k}^{t} f(x, t) d t
$$


Letting $\varepsilon \rightarrow 0$ shows that the integrated part vanishes. Hence the original problem leads to a two-point boundary-value problem

$$
\begin{aligned}
F_{t x x} & =a F_{x}+b F, \\
F(x, k) & =c \exp \left(-\int_{0}^{x}(b / a) d x\right), \\
F(x,-k) & =0 .
\end{aligned}
$$

Conversely, from such an $F$ we can construct, at least formally, a solution to the question first proposed.

Many of the foregoing considerations apply with only slight change to the equation

$$
\varepsilon y^{\prime \prime}+a(x) y^{\prime}+b(x) y=c(x) .
$$

The condition $f_{0}^{\prime \prime}=0$ in (26) is replaced by $f_{0}^{\prime \prime}=c(x)$, and we are led to consider $H^{k} p$ with $p=f_{0}$. Similarly, the condition $0=a y_{0}^{\prime}+b y_{0}$ in (20) becomes

$$
h(x)=a y_{0}^{\prime}+b y_{0}
$$

with corresponding change in the boundary condition for the associated partial differential equation. (The equation itself does not change.)

That there is always a solution regular in $\varepsilon$, for some $c(x)$, is evident when we take $y=1, c=b(x)$. Actually, one can find a $c(x)$ such that the regular solution depends on $\varepsilon$. For example, let $f$ satisfy

$$
f^{\prime \prime}+a f^{\prime}+b f=0, \quad f \approx 0,
$$

and let $c(x)=-f^{\prime \prime}(x)$. Then $y=f(x) /(1-\varepsilon)$ is a solution of (37).

5. A hydrodynamic application. Differential equations of the type (4) with

$$
M[y, \varepsilon]=x M^{*}[y, \varepsilon]
$$

where the leading coefficient $b_{0}{ }^{*}(x, 0)$ of $M^{*}[y, 0]$ does not vanish at $x=0$ occur in the theory of hydrodynamic stability. This application will be explained below. We shall be concerned here with necessary conditions on a differential equation (4), for which (38) is satisfied, in order that it possess a full contingent of $m$ solutions that converge to solutions of $M^{*}[y, 0]=0$, as $\varepsilon \rightarrow 0$, uniformly in a full neighborhood of $x=0$.

Before stating our theorem concerning this case we recall ([4], p. 126) that for linear differential expressions there exists division algo- 
rithm involving only rational operations and differentiations, by means of which $N[y, \varepsilon]$ can be represented in a unique fashion in the form

$$
N[y, \varepsilon] \equiv Q\left[M^{*}[y, \varepsilon], \varepsilon\right]+R[y, \varepsilon] .
$$

Here $Q[u, \varepsilon]$ and $R[y, \varepsilon]$ are linear differential expressions with $(x, \varepsilon)$ holomorphic coefficients. The order of $R[y, \varepsilon]$ is at most $m-1$. Let us call $R[y, \varepsilon]$ the remainder of $N[y, \varepsilon]$ with respect to $M^{*}[y, \varepsilon]$.

THEOREM 3. Assume that the differential equation

$$
\varepsilon N[y, \varepsilon]+x M^{*}[y, \varepsilon]=0
$$

possesses $m$ solutions of the form

$$
Y_{j}(x, \varepsilon)=y_{j 0}(x)+\varepsilon v_{j}(x, \varepsilon), \quad(j=1, \cdots, m)
$$

where the $y_{j 0}(x)$ form a fundamental system of the reduced equation

$$
M^{*}[y, 0]=0
$$

and the $v_{j}(x, \varepsilon)$ are bounded, together with their first $n$ derivatives with respect to $x$, at $x=0$, and for $\varepsilon$ in some point set $E^{*}$ having $\varepsilon=0$ as an accumulation point. Then the remainder $R[y, \varepsilon]$ of $N[y, \varepsilon]$ with respect to $M^{*}[y, \varepsilon]$ vanishes for $x=\varepsilon=0$, identically for all $y(x)$.

The conditions on $Y_{\jmath}(x, \varepsilon)$ in this theorem are much weaker than regularity. The meaning of Theorem 3 is essentially that even these weaker conditions will only exceptionally be satisfied, since for arbitrary $N[y, \varepsilon]$ and $M^{*}[y, \varepsilon]$ the remainder will, in general, not vanish identically in $y$, for $x=\varepsilon=0$.

Proof of Theorem 3. Without loss of generality we may assume that

$$
y_{j 0}^{(k-1)}(0)=\delta_{j k}, \quad(j, k=1, \cdots, m)
$$

If (39) is inserted in (40) and $y$ is replaced by $Y_{J}(x, \varepsilon)$, then use of (41) leads to a relation of the form

$$
\varepsilon^{2} \phi_{j}(x, \varepsilon)+\varepsilon x \psi_{j}(x, \varepsilon)+\varepsilon R\left[y_{j 0}, 0\right]=0, \quad(j=1, \cdots, m)
$$

where $\phi_{j}(0, \varepsilon)$ and $\phi_{j}(0, \varepsilon)$ remain bounded as $\varepsilon \rightarrow 0$ in $E^{*}$. Setting $x=0$ and letting $\varepsilon \rightarrow 0$ in $E^{*}$, this yields

$$
R\left[y_{30}, 0\right]=0, \quad \text { for } \quad x=0 \quad(j=1, \cdots, m) .
$$

Because of (43) we conclude that every coefficient of $R[y, 0]$ vanishes at $x=0$. This proves the theorem.

Application. By a simple change of variables the Orr-Sommerfeld equation in the theory of hydrodynamic stability, [5], 


$$
\underset{\alpha R}{i}\left[\frac{d^{\prime} \phi}{d z^{4}}-2 \alpha^{2} \frac{d^{2} \phi}{d z^{2}}+\alpha^{4} \phi\right]+(w(z)-c)\left(\frac{d^{2} \phi}{d z^{2}}-\alpha^{2} \phi\right)-w^{\prime \prime}(z) \phi=0
$$

can be written in the form

$$
\varepsilon\left(y^{(1)}-2 \alpha^{2} y^{\prime \prime}+\alpha^{1} y\right)+b_{0}(x)\left(y^{\prime \prime}-\alpha^{2} y\right)-b_{0}^{\prime \prime}(x) y=0
$$

with $b_{0}(0)=0$. (The dependence of $b_{0}(x)$ on the complex parameter $c$ is not set in evidence in our notation. The letter $\alpha$ denotes a positive constant.) The special case that $b_{0}^{\prime \prime}(x)$ also vanishes at $x=0$ is of some interest in hydrodynamics. If $c$ is real, for instance, and $b_{0}^{\prime \prime}(0)=0$, one has the case of a periodic disturbance of the flow such that the critical layer where the disturbance and the main flow travel with equal velocities, coincides with an inflection point of the main flow profile $w(z),[7]$. In the present case

$$
b_{0}(x)=\beta_{1} x+\beta_{3} x^{3}+\beta_{4} x^{4}+\cdots,
$$

and the remainder $R[y, \varepsilon]$ in Theorem 3 is independent of $\varepsilon$. A straightforward calculation, not reproduced here, shows that this remainder vanishes for $x=0$, if and only if

$$
\beta_{4}=0, \quad 3 \beta_{3}^{2}-5 \beta_{5} \beta_{1}=0 .
$$

Since the coefficients $\beta_{j}$ depend on $c$ these conditions can be satisfied for very exceptional profiles and very special disturbances only. Now, it is known, [14], that corresponding to every solution of the reduced equation there exist solutions of the full equation (45) having the form (41), with $v_{j}(x, \varepsilon)$ and its derivatives bounded in some region $S$ of the $x$-plane. As we have just seen, $S$ will not include the origin, at least not for all such solutions, unless very exceptional conditions are satisfied. From this it can easily be deduced that $S$ cannot be a doubly connected domain surrounding the origin completely, i.e., some solutions which converge in certain regions to a solution of the reduced equation, must diverge in certain other regions. It follows from this fact (cf. [14], [5]) that the damped disturbances of the corresponding hydrodynamic flow possess a so-called "inner friction layer," i.e., a layer in which the effects of viscosity cannot be neglected no matter how small the viscosity coefficient.

Thus Theorem 3 leads to the result that even if $w(z)-c$ and $w^{\prime \prime}(z)$ vanish at the same point for a certain damped disturbance, an inner friction layer will be present unless the disturbance and the velocity profile are of an extremely exceptional type.

It can be shown that the vanishing of $R[y, \varepsilon]$ at $x=\varepsilon=0$ is only one of infinitely many conditions necessary for the existence of $m$ regular solutions. It is therefore very likely, but not yet proved, 
that $b_{0}(x)=x$ (Couette flow) is the only flow for which inner friction layers are ever absent. In the Couette case the remainder $R[y, \varepsilon]$ is, of course, identically zero for all $x$ and $\varepsilon$, and every solution of the reduced equation is trivially a regular solution of the full equation.

\section{REFERENCES}

1. E. Hill, Lineare Differentialgleichungen im komplexen Gebiet. Enzykl. der Math. Wiss. II, 2, B, 5, Leibzig (1921).

2. J. Horn, Ueber lineare Differentialgleichungen mit einem veränderlichen Parameter. Math. Ann. 52 (1899), 340-362.

3. M. Hukuhara, Sur les propriétés asymptotiques des solutions d'un système d'équations differentielles linéaires contenant un paramètre. Mém. Fac. Engin. Kyushu Imp. Univ., Fukuoka, 8 (1937), 249-280.

4. E. L. Ince, Ordinary differential equations. Dover Publications, New York (First published 1926).

5. C. C. Lin, On the stability of two-dimensional parallel flows, part I. Quart. Appl. Math., 3 (1945) 117-142.

6. P. Noaillon, Dévéloppements asymptotiques dans les équations linéaires à paramètre variable. Mém. Soc. Roy. Sc. Liège, III e série, 9 (1912), 197.

7. W. Tollmien, Ein allgemeines Kriterium der Instabilität laminarer Geschwindigkeitsverteilungen. Nachr. Gesell. Wiss. Goettingen, VI (1935), 79-114.

8. W. J. Trjitzinsky, The theory of linear differential equations containing a parameter. Acta Math., 67 (1963), 1-50.

9. H. L. Turrittin, Asymptotic solutions of certain ordinary differential equations containing a parameter. Amer. J. Math., 58 (1936), 361-376.

10. - Asymptotic expansions of solutions of systems of ordinary differential equations. Contributions to the theory of nonlinear Oscillations, vol. II, 81-116, Princeton Univ. Press, 1952.

11. W. Wasow, Asymptotic solution of the differential equation of hydrodynamic stability in a domain containing a transition point. Ann. Math., 58 (1953), 222-252.

12. - A study of the differential equation $y^{(4)}+\lambda^{2}\left(x y^{\prime \prime}+y\right)=0$ for large values of $\lambda$. Ann. Math., 52 (1950), 350-361.

13. _- On small disturbances of plane Couette flow. J. Res. Nat. Bur. Stand., Sept. 1953.

14. The complex asymptotic theory of a fourth order differential equation of hydrodynamics. Ann. Math., 49 (1948), 852-871.

UNIVERSITY OF CALIFORNIA, LOS ANGELES

INSTITUTE FOR NUMERICAL ANALYSIS, LOS ANGELES 


\section{PACIFIC JOURNAL OF MATHEMATICS}

\section{EDITORS}

\author{
H. L. Royden \\ Stanford University \\ Stanford, California \\ E. Hewite \\ University of Washington \\ Seattle 5 , Washington
}

\author{
R. P. Dilworth
}

California Institute of Technology Pasadena 4, California

\section{A. HorN*}

University of California

Los Angeles 24, California

\section{ASSOCIATE EDITORS}

\author{
H. BUSEMANN \\ HERBERT FEDERER \\ MARSHALL HALL
}

\author{
P. R. HALMOS \\ HEINZ HOPF \\ ALFRED HORN
}

\author{
R. D. JAMES \\ BORGE JESSEN \\ PAUL LÉVY
}

GEORGE PÓLYA

J. J. STOKER

KOSAKU YOSIDA

\section{SPONSORS}

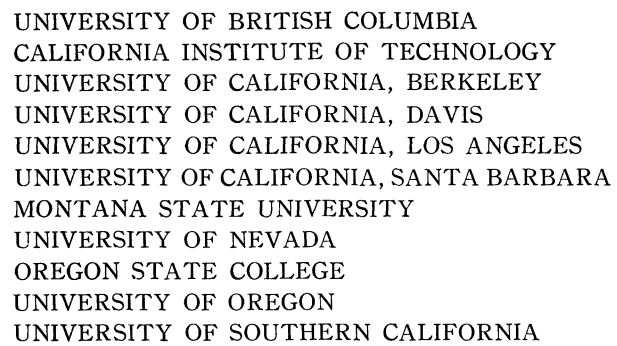

UNIVERSITY OF BRITISH COLUMBIA

CALIFORNIA INSTITUTE OF TECHNOLOGY

UNIVERSITY OF CALIFORNIA, BERKELEY

UNIVERSITY OF CALIFORNIA, DAVIS

UNIVERSITY OF CALIFORNIA, LOS ANGELES

UNIVERSITY OF CALIFORNIA, SANTA BARBARA

MONTANA STATE UNIVERSITY

UNIVERSITY OF NEVADA

OREGON STATE COLLEGE

UNIVERSITY OF OREGON

UNIVERSITY OF SOUTHERN CALIFORNIA

\author{
STANFORD RESEARCH INSTITUTE \\ STANFORD UNIVERSITY \\ UNIVERSITY OF UTAH \\ WASHINGTON STATE COLLEGE \\ UNIVERSITY OF WASHINGTON
}

AMERICAN MATHEMATICAL SOCIETY

HUGHES AIRCRAFT COMPANY SHELL DEVELOPMENT COMPANY

Mathematical papers intended for publication in the Pacific Journal of Mathematics should be typewritten (double spaced), and the author should keep a complete copy. Manuscripts may be sent to any of the editors. Manuscripts intended for the outgoing editors should be sent to their successors. All other communications to the editors should be addressed to the managing editor, Alfred Horn at the University of California Los Angeles 24, California.

50 reprints of each article are furnished free of charge; additional copies may be obtained at cost in multiples of 50 .

The Pacific Journal of Mathematics is published quarterly, in March, June, September, and December. The price per volume (4 numbers) is $\$ 12.00$; single issues, $\$ 3.50$; back numbers (Volumes $1,2,3)$ are available at $\$ 2.50$ per copy. Special price to individual faculty members of supporting institutions and to individual members of the American Mathematical Society: $\$ 4.00$ per volume; single issues, $\$ 1.25$.

Subscriptions, orders for back numbers, and changes of address should be sent to the publishers, University of California Press, Berkeley 4, California.

Printed at Kokusai Bunken Insatsusha (International Academic Printing Co., Ltd.) No. 10 1-chome Fujimi-cho, Chiyoda-ku, Tokyo, Japan.

* During the absence of E. G. Straus. 


\section{Pacific Journal of Mathematics}

\section{Vol. 5, No. $5 \quad$ BadMonth, 1955}

Henry A. Antosiewicz, A theorem on alternatives for pairs of matrice . . . . . 641

F. V. Atkinson, On second-order non-linear oscillation ............... 643

Frank Herbert Brownell, III, Fourier analysis and differentiation over real separable Hilbert spac .................................. 649

Richard Eliot Chamberlin, Remark on the averages of real function ...... 663

Philip J. Davis, On a problem in the theory of mechanical quadrature ... . . 669

Douglas Derry, On closed differentiable curves of order $n$ in $n$-spac ...... 675

Edwin E. Floyd, Boolean algebras with pathological order topologie ... . . 687

George E. Forsythe, Asymptotic lower bounds for the fundamental frequency of convex membrane ................................. 691

Israel Halperin, On the Darboux propert ................... 703

Theodore Edward Harris, On chains of infinite orde .............. 707

Peter K. Henrici, On certain series expansions involving Whittaker functions

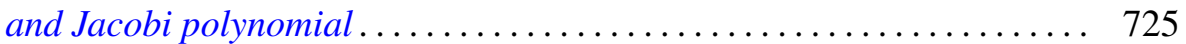

John G. Herriot, The solution of Cauchy's problem for a third-order linear hyperoblic differential equation by means of Riesz integral ......... 745

Jack Indritz, Applications of the Rayleigh Ritz method to variational

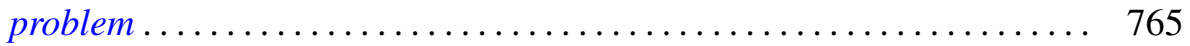

E. E. Jones, The flexure of a non-uniform bea ................. 799

Hukukane Nikaidô and Kazuo Isoda, Note on non-cooperative convex game.

Raymond Moos Redheffer and W. Wasow, On the convergence of asymptotic solutions of linear differential equation . . .

S. E. Warschawski, On a theorem of L. Lichtenstei ...........

Philip Wolfe, The strict determinateness of certain infinite game... 